\title{
The Extrinsic and Intrinsic Factors Predisposing to ACL Injuries \\ in Female Athletes - Sports Medicine Implications in 2021
}

\author{
Alexander Mitrichev ${ }^{1}$, Rami Shenouda ${ }^{2}$ and Mark Daniel Wilson ${ }^{3 *}$ \\ ${ }^{1}$ Orthopaedic Registrar, Cairns Base Hospital, QLD, Australia \\ ${ }^{2}$ Emergency Medicine Registrar, Alfred Health, Victoria, Australia \\ ${ }^{3}$ Sport and Exercise Medicine Registrar, Qsports Medicine, QLD, Australia \\ *Corresponding Author: Mark Daniel Wilson, Sport and Exercise Medicine \\ Registrar, Qsports Medicine, QLD, Australia.
}

Received: March 23, 2021

Published: April 20, 2021

(C) All rights are reserved by Mark Daniel

Wilson., et al.

\begin{abstract}
Anterior cruciate ligament (ACL) injury is one the most common knee injuries in the young and middle age population. Large numbers of studies have examined the anatomy, biomechanics, management and rehabilitation of ACL injuries in the general population and athletes. Contemporary medical technology has facilitated new treatment paradigms and lead to the exponential growth in knowledge in this fundamental area of knee surgery and sports medicine. The objective of this narrative review was to identify studies that examined certain intrinsic and extrinsic factors contributing to ACL injury risk in female athletes. Participation in sports by females is increasing globally and the burden of disease in relation to ACL injuries is expected to increase in association with these increased participation rates.

There is a paucity of literature that clearly delineates the intrinsic and extrinsic risk factors for ACL injury among different populations, especially higher-level female athletes. To facilitate the best possible care for knee injured patients, it is essential to understand the key factors that may underpin different injury patterns, especially in the context of dramatically increased participation in high risk sports, including Australian Rules Football, rugby union and rugby league, basketball, netball, soccer and skiing; focusing on those identifiable risk factors that have a female predilection will be the focus of this review and an attempt has been made to broadly review the most pertinent scientific literature.
\end{abstract}

Keywords: ACL Injury; Female ACL; ACL Risk Factors; ACL and Proprioception

\section{Introduction}

The anterior cruciate ligament (ACL) is one of the two major intra-articular ligaments of the knee joint that is crucial for anteroposterior stability and sagittal plane constraint during knee flexion and extension [1]. Disruption of the ACL can lead to significant morbidity and best practice management, regardless of whether surgery or conservative measures are undertaken, requires pro- longed rehabilitation; the ACL-deficient knee is also at increased risk of developing premature osteoarthritis (OA) over time. It has been postulated that knee OA develops as a result of altered or decreased proprioception secondary to ACL deficiency. It may be the case that ACL deficiency leads to alterations in joint loading characteristics and ongoing, sustained chondrocyte injury [2,3]. Due to specific anatomical and biomechanical properties, the ACL

Citation: Mark Daniel Wilson., et al. "The Extrinsic and Intrinsic Factors Predisposing to ACL Injuries in Female Athletes - Sports Medicine Implications in 2021". Acta Scientific Orthopaedics 4.5 (2021): 11-19. 
accounts for 40 - 50\% of all knee ligament injuries in people participating in recreational sport, as well as professional athletes(3). The ACL injury risk factors were divided into intrinsic and extrinsic components.

\section{Extrinsic factors}

Types of sporting activity

Australia has the highest number of ACL injuries in the world. The majority of ACL ruptures can be observed in groups of people joining recreational and professional sport. A large systematic review and meta-analysis stratified sports into collision, contact, limited contact, non-contact and fixed object high-impact rotational landing (HIRL) [4]. The high-impact rotational landing group was created after sports deemed non-contact produced several outlier results post analyses. The incident rates ratios (IRR) for ACL injury for females in comparison to males were both largest and statistically significant in the contact and fixed-object HIRL sports, with the IRR of 3.0 , (95\% CI 2.70 to $3.34, \mathrm{P}<0.001)$ and $5.51(95 \%$ CI 2.80 to $10.82, \mathrm{P}<0.001$ ), respectively. Despite only the contact and fixed-object HIRL sports having statistically significant results, within each sporting group, females demonstrated higher rates of ACL injury, compared to their male counterparts, with all other factors controlled for.

\section{Australian rules football}

Female participation in Australian Rules Football (AFL) has increased significant in recent years, largely due to the creation of the AFLW professional league and many more reserve level and amateur grade football clubs all across Australia. Female involvement in AFL increased by 21,000 from December 2017 to December 2018 [5], with an estimated 530,166 females playing across Australia; these numbers are expected to grow in coming years as the game becomes more popular among younger and more diverse groups nationally. The annual incidence growth of ACL injury in the Australian population increased by $3.8 \%$ and $1.7 \%$ for under 25-year-olds and above 25-year-olds, respectively [6]. Indeed, AFL causes the highest rate of ACL injury among all sport in Australia, making female players from 6.2 to 9.2 times higher risk compared with male counterparts [7]. Comparing similar sports overseas, it was found that males were four times more likely to sustain an ACL injury whilst playing American football (Gridiron) compared to any other sport (RR 4.0,95\% CI 3.20 to 5.03) in botl collegiate and national level competitions [8].

\section{Rugby codes}

ACL injury is common in Rugby games with an identified injury rate of 0.43 per 1000 player hours. Despite general believe that non-contact mechanisms contribute to the majority of cases, two noticeable studies proposed the opposite view $[9,10]$. Authors found that $57 \%$ of injuries occurred after contact with other players, where offensive playing situation were recorded in more than $60 \%$ of all contact ACL tears. Furthermore, no significant difference was found in knee injuries between Australian Rugby Union and Rugby League. However, no regression analysis was performed to define whether this trend would be seen in ACL injury. Incidence rate (IR) of ACL injury in female rugby players is 5.3 (95\% CI: 1.03, 7.52) times higher compared with male athletes, but lower that IR of ACL rupture in female AFL players [11].

\section{Soccer}

Soccer is one of the most popular sports in Australia. In the US, female soccer players suffer from ACL injuries more often than players of other sports. Stanley., et al. (2016) found that the rate of ACL injury was as high as 2.55/10000 at the collegiate level. This number was even higher (3.2/10000) from both contact and non-contact mechanisms and irrespective of age in other studies. Further analysis of the literature also suggested that this figure is 2 - 3 times lower in males [12]. However, it is unclear whether or not these results can be extrapolated to all age groups of soccer players.

\section{Basketball}

More than one million Australians play basketball. However, limited data is available in Australia literature regarding the incidence of ACL injury in basketball players. According to studies from the US, for all knee injuries, ACL injury in female players demonstrated the greatest sex difference at the high school level (3.68 95\% CI 1.91 - 7.10) when stratified by sport; however, the former also had a significantly large confidence interval. The study demonstrated that, while there were sex-based differences in ACL injury rate, there were no overall differences in rate ratio in medial collateral ligament (MCL) and meniscal injuries. However, the researchers were unable to determine whether injuries occurred concomitantly or independently $[12,13]$.

Skiing

A previous retrospective study looking at all injuries in World Cup (elite) skiers found no statistical difference in ACL injury rate 
between male and female skiers (Relative Risk 1.03 95\% CI, 0.68 to 1.58) over the course of six consecutive winter seasons. When only World Cup race numbers were analysed, again, no difference was identified. A subsequent study by the same group into elite skiers also found no difference in ACL injury risk between the sexes; however, this study identified that injury rate across disciplines of skiing increased with increasing speed, which may explain why men historically sustained a higher rate of ACL injuries in the more elite levels of competition (RR 1.24 95\% CI 1.05 to 1.47 [14].

\section{Duration of sporting activity}

Female athletes have 1.5 times greater risk of ACL rupture from first season to 25 years in all sports. Incidence of ACL injury is also higher in younger females by $70 \%$, which equated to one injury in 29 female athletes compared to one in 50 in their male conterparts. The pooled ACL injury incidence proportion for all athletes was $2.8 \%$ (95\% CI 2.4 to $3.3 \%$ ) [15].

Specifically for soccer group participants from the first season to four years, absolute risk for ACL injury was 2.2 higher in females. Interestingly, when accounting for participation level, the difference between sexes was found to be the greatest for intermediate players ( IRR = 2.9, 95\% CI $2.4-3.6$ ) in comparison to beginners ( $\mathrm{IRR}=2.6$; $95 \%$ CI 1.4 to 4.8 ) and elite players (IRR $=2.0 ; 95 \%$ CI 1.1 to 3.4 ).

\section{Playing surface and shoes}

Male cohorts exhibited no difference in ACL injury rate between the two playing surfaces (natural and artificial). However, females had an increased risk by 2.4 times if participating in a $\mathrm{Eu}-$ ropean handball game on an artificial surface [16]. Other group o* researchers also reviewed shoe factors. The results demonstratec that shoes with small peripherally placed cleats or pointing cleat: positioned internally have the highest risk for ACL injury. The com bination of grass shoe and grass surface may be the optimal on in the prevention of injury; however, it is not an independent risl factor. The same authors investigated the role of knee bracing. Thi: result was inconclusive [17].

\section{School vs. collegiate factors}

Paralleling the rise in adult-onset ACL injuries, the paediatric and adolescent population demonstrated an almost a $150 \%$ increase in ACL injury rate in the ten years prior to 2015 , with sportrelated ACL rupture more than $50 \%$ from those females contribut- ing to more than half of all cases [6]. One study collected ACL injury data from eight colleges and 18 high schools across seven sports over a four-year period. College athletes reported 48 ACL injuries across all sports (IR $=0.150$ per 1000 person-days), in comparison to the 53 ACL injuries (IR $=0.061$ per 1000 person-days) in high schools. Overall, females were more than twice as likely to have a first-time non-contact ACL injury, with a relative risk of 2.1 (95\% CI, 1.34 to 3.27 ). College athletes had a higher injury risk than high school athletes post adjustment, with a relative risk of 2.38 (95\% CI, 1.55 to 3.64). The study was not without limitations. Measurement of athlete exposure was not as exact as desired by the authors. Despite this, the study remained of strong quality and had a larger size than previous cohort studies, giving credence to the conclusions [18].

\section{Parental ACL injury}

Other recent studies have aimed to identify factors that predispose female athletes to ACL injuries in more general terms. Parent with an ACL injury history is the strongest predictor of ACL rupture, especially for females with OR 3.8 - 3.84. However, this finding is statistically significant in combination with other intrinsic factors, such as increase AP knee displacement ( $\mathrm{mm})$, increased trunk flexion strength (Nm) and higher BMI. Univariate analysis also identified that navicular drop and change in hip flexion and trunk flexion were associated with an increase odds ratio of ACL injury. A decrease in passive genu recurvatum and hip adduction were also associated with increased risk of ACL injury.

\section{Intrinsic factors}

\section{Tibial properties}

The first study reviewed demonstrated that for each degree increase in slope of the lateral tibial plateau, there was a $21.7 \%$ increase risk of non-contact rupture of the ACL. On initial review of the data from one cohort study, it suggested that there was an $14.6 \%$ increase in risk of ACL rupture in all participants; however, when participants were separated by sex, there was no discernible risk in males, whereas the risk for females was much more pronounced [20].

Another study found that females with injured ACLs had increased lateral tibial slope (8.44 vs. 7.03 degrees) and shallower medial tibial depth (1.91 vs. $2.75 \mathrm{~mm}$ ) compared to the uninjured female controls, where males had both increased medial and lat- 
eral tibial slope, as well as shallower medial depth. The study's pooled data suggested that medial tibial depth is an important risk factor (odds ratio $=3.03$ per $1 \mathrm{~mm}$ decrease in value), followed by lateral tibial slope (odds ratio $=1.17$ per 1 degree increase in value) for all participants, as well as medial tibial slope (odds ratio 1.18 per 1 degree increase in value) for males only, making overall odds ratio higher when these factors were included together (3.58 in females and 4.18 in males). The study also could not establish any difference in the variables between injured males and females, but they shared similar high-risk predisposing factors. Whilst also a case-control study, there is potential bias from MRI interpretation and the lack of matching in relation to height, weight and exposure to activities that could place participants at higher risk is a weakness of this study [21].

\section{Size of the femoral notch}

Femoral notch size has been significantly reviewed as a contributing factor in ACL injury, specifically for female athletes. Multiple papers concluded that a narrow femoral notch was strongly associated with an increased risk of ACL rupture, as an independent, anatomical risk factor. Based on that data, we concluded that as females are generally smaller than males, that the femoral notch would also be smaller. The assumption that females have smaller notches and ligaments (in cross-section) has also been described in standard orthopaedic and anatomical texts [21,22].

\section{Intercondylar width and alpha angle}

One of case-control studies attempted to address the importance of the intercondylar notch as the risk in ACL injury. The study had 508 participants, 308 with ACL rupture and 222 with normal ACLs and compared both the intercondylar notch width and the alpha angle (the angle between the long axis of the femur and Blummensaat's line) of the knee. The intercondylar width was $18.2 \pm 3.1$ $\mathrm{mm}$ vs $19.5 \pm 3.6 \mathrm{~mm}$ in those with a torn ACL compared to a normal ACL, in addition to a significant difference between the width of male and female subjects $(19.3 \pm 3.3 \mathrm{~mm}$ vs. $17.4 \pm 3.1 \mathrm{~mm})$. This study also demonstrated that there were, in the individuals with torn ACLs, slightly increased alpha angles ( $57.5 \pm 5.5 \mathrm{~mm}$ vs. $56.2 \pm$ $4.5 \mathrm{~mm}, \mathrm{P}=0.009$ ). The researchers hypothesised that this related to increased impingement in extension that resulted in more frequent ACL tears. Finally, there were an increased number of ACL tears in the male subjects enrolled in the study, however the significance of this is unclear [22].

\section{Femoral notch in skeletally immature patients}

Femoral notch size has also been reviewed in the skeletally immature group. A review investigated notch width index (intercondylar notch width/bicondylar femoral width) as a predictor for non-contact ACL injury in the skeletally immature patient [23]. After initially finding 128 candidates, 39 skeletally immature individuals with ACL injury (average age 14) were age-matched in a case-control study. Individuals with isolated ACL injuries demonstrated decreased notch width index, however further logistic regression analysis was not predictive of injury. The combined ACL/ MCL subgroup had statistically significant differences with large intercondylar notch volumes, smaller tibial eminence volume, smaller medial tibial plateau slope and depth and smaller lateral plateau depth when compared to the control group. Finally, on further analysis, there were significant differences based on gender, with female patients having decreased intercondylar notch volume, tibial eminence height, width and volume. While this study was conducted over a significant time period, the study itself was small, with 89 potential candidates excluded for a variety of reasons which may have limited its strength. A single radiologist performed the measurements to reduce variability in measurement. Prior to this, there were limited studies into the skeletally immature patient with an ACL injury [23].

\section{ACL characteristics}

The evidence pertaining to ACL size and the difference between male and female ACL morphology and injury rates is conflicting. Recent literature has aimed to build on the view that females have smaller ACLs in general (width on axial and sagittal views, as well as total area), in comparison to males [24] and consequently were more likely to have ACL injuries. ACL injuries were due to a complex interaction of extrinsic and intrinsic factors and did not solely identify the ACL properties above as the sole critical differences [24]. The subsequent studies that have built on this have generally reviewed ACL parameters and volume in conjunction with notch properties [25]. Is the ACL more than just an end-range restrictor or is it an organ of proprioception? These considerations will be examined later in this review, but much work remains to be done in the field of knee surgery and sports medicine to answer fundamental questions of clinical importance.

\section{ACL volume}

Research in 2009 suggested that the female participants had statistically significant decrease in mean ACL volume by $185.8 \mathrm{~mm}^{3}$ 
(injured vs. control, 1694.5 and $1880.3 \mathrm{~mm}^{3}, \mathrm{p}$ 0.0115) compared to the male participants, whose difference was only $149.1 \mathrm{~mm}^{3}$ (injured vs. control, 2107.5 and $2256.5 \mathrm{~mm}^{3}$, p 0.2149) [26]. However, once stepwise regression was performed on the data, only weight was found to be a significant covariate, whereas gender, height and age were not, once adjusted for it. The study also highlighted some key weaknesses, such as manual segmentation of the ACL from the MRI due to difficulties in visualising key structural differences, as well as the use of ACL volume rather than cross-sectional area. The latter comment is due to stress across a ligament being equal to force divided by cross-sectional area, however the author comments that volume should be a suitable surrogate marker, assuming a typical, homogenous ACL morphology [26].

\section{ACL length and width}

Increased length of ACL anterior $(\mathrm{P}<0.01)$ and posterior edge $(\mathrm{P}<0.01)$ and decreased ACL width $(\mathrm{P}<0.05)$ in an MRI-based case-control study is associated with non-contact ACL injuries [27]. There was a direct correlation with femoral intercondylar notch. No conclusion was drawn on the significance of this connection nor on gender specific variation of results [27]. Further investigating these characteristics using sex variables, research quantified notch width as a measure of ACL width and volume, rather that ACL length or width. Decreased ACL volume with a combined OR 0.829 was a risk factor for ACL injury, with a more pronounced effect in males. At the same time, decreased femoral notch width with OR 0.7 had a strong correlation with injury in the female group of participants. Increased thickness of the bony ridge at the anteromedial outlet of the femoral notch (OR 1.614) was another independent risk factor, with similar distribution in both sexes. Overall, this study did contribute to the hypothesis that parameters of ACL and femoral notch are key anatomical contributors in ACL injury.

\section{Q-angle}

Generally, females have increased Q-angle of the lower limbs compare with male counterparts (26). A larger Q-angle leads to a superolateral vector, which tends to pull on the patella in all functional planes of motion, but has the most significance in knee flexion less than $30 \mathrm{deg}$ and in terminal extension, when the contact pressures on the femoral trochlear and lateral facet of the patella are reduced; it is unclear whether this places more strain on the ACL and further novel biomechanics-based studies, such as finite element analysis modelling, could help answer this question.
Knee and generalised ligamentous laxity

Laxity has been described in the literature as a potential contributor to ACL injury in males. As force passes through the knee, the hamstrings and quadriceps isometrically contract, thereby protecting the ipsilateral ACL. In cases of knee laxity, this protective, well-coordinated mechanism is absent or there is an imbalance between hamstring or quadriceps tonic contraction or rate and timing or activation. To disseminate the imparted energy, the knee transmits force in three directions by causing hyperextension, hypervalgus and increase anterior translation of the tibia in respect to the femur [28]. The anatomy of the female knee demonstrated a statistically significant increase in anterior tibial translation difference $(6.05 \pm 1.46 \mathrm{~mm}$ in comparison to $\mathrm{men}=4.80 \pm 1.53 \mathrm{~mm})$. It is thus difficult to generalise whether these disparity would still exist in individuals with ACL injuries or if such a difference would be reduced or magnified in certain athletic populations [29].

Following this, another paper added to the topic of laxity by demonstrating significant associations between ACL injury history and generalised ligamentous laxity, tibia varum, high Q-angle, genu recurvatum, femoral anteversion, dorsiflexion range of motion, hamstring flexibility, ITB flexibility, hip flexor flexibility, foot type, and ankle flexibility [30]. A discriminant regression model demonstrated that the strongest relationships were for generalised laxity $\left(r^{2}\right.$ change 0.073$)$, genu recurvatum $\left(r^{2}=0.069\right)$ and ITB flexibility $\left(r^{2}=0.069\right)$. A further stepwise linear regression demonstrated that each of these three factors were statistically significant contributors to ACL injury in the female athlete. The importance of laxity may also be strengthened as genu recurvatum is a term used for hyperextension of the knee [30]. Genu recurvatum had previously been implicated in ACL injury rate, where it was found to have a significant association in combination with subtalar joint overpronation, whilst hyperextension of the knee joint has been implicated in lower limb injuries more broadly.

\section{Limb dominance}

In previous reviews, limb dominance has been included in the intrinsic factors that may affect ACL injury rate [4]. Most research has been conducted on soccer players. An RCT by of 4556 teenagers showed the incidence of ACL injury in non-dominant limbs vs. dominant limbs in female soccer players: $11 / 21$ (52\%) vs. 6/21 (29\%) [24]. Similarly, other study found that only $32 \%(\mathrm{P}<0.002)$ of female ACL injuries were associated with the dominant limb and 
was more commonly associated with non-contact patterns of injury [25]. Last but not least, a retrospective review of 80 patient also showed the small prevalence of non-dominant ACL injury in $54 \%$ of cases, with $89 \%$ of players self-identifying as right leg kickers [26].

Conversely, male players had a notably inverse proportion in dominance of injury [25]. A study which focused on elite level female athletes did not confirm correlation with dominance and ACL rupture in contact soccer injury [27]. Reviewing recreational skiing, the trend was similar and demonstrated a 2-fold increase of ACL rupture on the non-dominant limb in 193 recreational skiers. Non-dominant ACL injury had a univariate odds ratio of 2.1 (95\% CI, 1.1 to 3.8, P 0.020 ) and a multivariate odds ratio of 2.00 (95\% CI 1.00 to 3.78, P 0.049) when comparing female and male participants. Cumulatively, however, the study supported the legdominance theory, albeit in the recreational female athlete cohort. It was concluded that non-elite level female athletes had a higher incidence of non-dominant ACL injury in non-contact settings, whereas males and elite female athletes may demonstrate better limb balance and relative symmetry in the distribution of ACL injury patterns.

\section{Avenues for future research}

At present, surgical reconstruction of the ACL is a mechanical solution to a mechanical problem. The increase in both objective and subjective knee instability in patients with ACL-deficient knees can be addressed with surgical reconstruction, but surgically optimal graft placement and superior technique do not necessarily confer a restoration to pre-injury objective and subjective function [28]. We propose that the anterior cruciate ligament is an organ of proprioception, whose importance has not been sufficiently appreciated. Indeed, the preservation of the tibial footprint of the native ACL at the time of ACL reconstruction surgery, in an effort to preserve mechanoreceptors to augment the functional benefits of ACL reconstruction, insofar as it doesn't compromise graft placement, has been practiced for many years [29].

It has been theorised that the arthritis observed in the ACL-deficient knee joint develops or is accelerated as a result of altered or decreased proprioception [30]. It may be the case that ACL deficiency leads to alterations in joint loading characteristics and a potentiation of hyaline articular cartilage injury at the cellular level, especially in the setting of recurrent instability episodes; however, the harbinger of OA may in fact be the index pivot shift translation, which irreversibly damages the knee as a functional unit. The classical view of the ACL is that it is primarily an end-range restrictor; this view is well established, both theoretically and in practical terms [31]. The ACL has two functional bundles measuring a combined $3.2 \mathrm{~cm}$ length $x 0.7-1.2 \mathrm{~cm}$ width, on average; the lack of size of the ACL and most other ligaments do not support their role as a purely end range restrictor, but rather a 'mechanoreceptor vessel' that informs the rest of the kinetic chain to protect the limb as a whole from dangerous positions, whilst providing a degree of anteroposterior and rotatory stability to translation through traditionally conceived means [32].

Many biomechanical studies have shown considerable strength of the native ACL ( $2200 \mathrm{~N}$ to failure in anterior tibial translation), as well as the strength of allograft/autograft reconstructed ACLs (approaching 85-90\% of pre-injury levels at 9 - 12 months post-operatively), which are strong enough to withstand the physiologic loads placed on them during activities of daily living and sporting activities [33]. No biologic tissue is able to fully prevent injury when extreme stresses are placed through it. The intrinsic proprioceptive and mechanoreceptive properties of ligaments play an important and incompletely understood role in normal joint function. It is known that normal knee kinematics do not completely return after ACL reconstruction, placing additional strains on secondary stabilizers of the knee, including the meniscus, capsule, iliotibial band and MCL [34]. The kinematic alteration has been postulated to be a factor explaining the observation that knee OA rates are not significantly different in ACL reconstructed knees and in chronic ACL deficient knees, but it is unknown whether there are significant or meaningful gender differences in this observation [35]. Some studies have even suggested lower rates of OA in non-reconstructed knees [36].

Whether or not improved outcomes (namely, return to sport, lack of subjective instability, less functional instability episodes or improved objective measures of sagittal and rotatory plane stability, e.g. KT1000 measures) is more likely a result of the mechanical constraints of the graft than any contribution from any ingrowth of native proprioceptive nerve fibres has not been clearly established in the knee surgery and sports medicine literature. The dynamic relationship between mechanical stability and proprioceptive 
feedback control suggests that graft neuroregeneration may be an important adjunct to treatment as surgical and rehab protocols continue to improve [37]. The complex interplay between graft soft tissue integrity over time and restoration of proprioceptive feedback to the whole 'knee organ' warrants theoretical and clinical attention.

\section{Perspective}

There is gender-specific differences in ACL injury rates which have implications in relation to prognosis and outcome in athletes. Using extrinsic factors from this study, we propose that injury rates and the associated treatment costs can be reduced. Moreover, some important intrinsic factors may be used for routine assessment before entering in high-risk sports, especially for young athletes. Using multiple ACL variables, clinicians may create a tool which will estimate ACL injury risk. As the next step, data can be used in artificial intelligence (AI) to generate the "safest model" of playing sport to avoid knee injuries where applicable or provide an open statement about risks to particular individuals in their sporting pursuits.

\section{Conclusion}

There has been a significant amount of research completed on the background, aetiology, prevention and management of the rupture of the ACL in the sporting population. With time, there has been a significant volume of data related to the female athlete and the assessment of gender differences, but much work remains to be done to clarify the 'state of play'. While initial views suggest that the female athlete is 2 - 8 times more likely to rupture her ACL than her male counterpart, the data examined in this review suggests this number has many possible causes and confounders. The role of gender-based anatomical differences in relation to ACL injury continues to be evaluated. While recent studies on anatomical differences have suggested a number of reasons for an increase in the risk of ACL injury, a majority of these occur in both males and females.

\section{Ethics Statement}

this article is the second source of information. In-text citation and reference list are included. This study required no special funding for its design or construction.

\section{Informed Consent}

Not applicable.

\section{Conflict of Interest}

No conflict of interest.

\section{Bibliography}

1. Gwathmey FW and Burrus MT. "Anatomy: Section 3 Lower Extremity”. [Book auth.] Miller, M.D. and Thompson, S.R. Miller's Review of Orthopaedics. Philadelphia: Elsevier (2016).

2. Kweon C., et al. "Anatomy and biomechanics of the cruciate ligaments and their surgical implications". In The multiple ligament injured knee (2013): 17-27.

3. Thompson SR and Miller MD. "Sports Medicine: Section 1 Knee”. [book auth.] Miller, M.D. and Thompson, S.R.. Miller's Review of Orthopaedics. Philadelphia: Elsevier (2016).

4. Montalvo AM., et al. "Anterior Cruciate Ligament Injury Risk in Sport: A Systematic Review and Meta-Analysis of Injury Incidence by Sex and Sport Classification". Journal of Athletic Training (2019): 472-482.

5. Carney S. "Roy Morgan Research: AFLW Inspiring Female Sport Participation". Ministry of Sport (2019).

6. Shaw L and Finch CF. "Trends in Pediatric and Adolescent Anterior Cruciate Ligament Injuries in Victoria, Australia 20052015". International Journal of Environmental Research and Public Health 14 (2017): E599.

7. Fox A., et al. "Anterior cruciate ligament injuries in Australian football: should women and girls be playing? You're asking the wrong question". BMJ Open Sport and Exercise Medicine 6 (2020).

8. Montalvo AM., et al. "What's my risk of sustaining an ACL injury while playing sports?', A systematic review with meta-analysis". British Journal of Sports Medicine (2019): 1003-1012.

9. Montgomery C., et al. "Mechanisms of ACL injury in professional rugby union: a systematic video analysis of 36 cases". British Journal of Sports Medicine 52 (2016): 994-1001.

10. Awwad GEH., et al. "An Analysis of Knee Injuries in Rugby League: The Experience at the Newcastle Knights Professional Rugby League Team". Sports Medicine - Open 5 (2019).

Citation: Mark Daniel Wilson., et al. "The Extrinsic and Intrinsic Factors Predisposing to ACL Injuries in Female Athletes - Sports Medicine Implications in 2021". Acta Scientific Orthopaedics 4.5 (2021): 11-19. 
11. Peck KY., et al. "The Incidence of Injury Among Male and Female Intercollegiate Rugby Players". Sports Health: A Multidisciplinary Approach 5 (2013): 327-333.

12. Stanley LE., et al. "Sex Differences in the Incidence of Anterior Cruciate Ligament, Medial Collateral Ligament, and Meniscal Injuries in Collegiate and High School Sports: 2009-2010 Through 2013-2014". The American Journal of Sports Medicine (2016): 1565-1572.

13. Arendt EA., et al. "Anterior Cruciate Ligament Injury PatternsAmong Collegiate Men and Women". Journal of Athletic Training (1999): 86-92.

14. Bere T., et al. "Sex differences in the risk of injury in World Cup alpine skiers: a 6-year cohort study". Britich Journal of Sport Medicine 48 (2013): 36-40.

15. Joseph AM., et al. "A Multisport Epidemiologic Comparison of Anterior Cruciate Ligament Injuries in High School Athletics". Journal of Athletic Training 48 (2013): 810-817.

16. Sutton KM and Bullock JM. "Anterior Cruciate Ligament Rupture: Differences Between Males and Females". Journal of the American Academy of Orthopaedic Surgeons 21 (2013): 41-50.

17. Giugliano DN and Solomon JL. "ACL Tears in Female Athletes". Physical Medicine and Rehabilitation Clinics of North America 18 (2007): 417-438.

18. Beynnon BD., et al. "The Effects of Level of Competition, Sport, and Sex on the Incidence of First-Time Noncontact Anterior Cruciate Ligament Injury". The American Journal of Sports Medicine (2014): 1806-1812.

19. Vacek PM., et al. "Multivariate Analysis of the Risk Factors for First-Time Noncontact ACL Injury in High School and College Athletes: A Prospective Cohort Study with a Nested, Matched Case-Control Analysis". The American Journal of Sports Medicine (2016): 1492-1501.

20. Beynnon BD., et al. "Increased Slope of the Lateral Tibial Plateau Subchondral Bone Is Associated with Greater Risk of Noncontact ACL Injury in Females but Not in Males: A Prospective Cohort Study with a Nested, Matched Case-Control Analysis". The American Journal of Sports Medicine 42 (2014): 10391048.
21. Hashemi J., et al. "Shallow Medial Tibial Plateau and Steep Medial and Lateral Tibial Slopes : New Risk Factors for Anterior Cruciate Ligament Injuries". The American Journal of Sports Medicine 38 (2010): 54-62.

22. Fernández-Jaén T., et al. "The Importance of the Intercondylar Notch in Anterior Cruciate Ligament Tears". American Orthopaedic Society of Sports Medicine 3 (2020).

23. Shaw KA., et al. "Knee morphometric risk factors for acute anterior cruciate ligament injury in skeletally immature patients". Journal of Children's Orthopaedics 9 (2015): 161-168.

24. Hägglund M and Waldén M. "Risk factors for acute knee injury in female youth football". Knee Surgery, Sports Traumatology, Arthroscopy 24.3 (2016): 737-746.

25. Ruedl G., et al. "Leg Dominance Is a Risk Factor for Noncontact Anterior Cruciate Ligament Injuries in Female Recreational Skiers". The American Journal of Sports Medicine 40 (2012): 1269-1273.

26. Ajit MWC., et al. "ACL-Injured Subjects Have Smaller ACLs Than Matched Controls: An MRI Study". The American Journal of Sports Medicine 37 (2009): 1282-1287.

27. Stijak L., et al. "Morphometric parameters as risk factors for anterior cruciate ligament injuries - A MRI case-control study". Vojnosanit Pregl 71 (2014): 271-276.

28. Beynnon BD. "Relationship Between the Risk of Suffering a First-Time Noncontact ACL Injury and Geometry of the Femoral Notch and ACL: A Prospective Cohort Study with a Nested Case-Control Analysis". The American Journal of Sports Medicine 42 (2014): 1796-1805.

29. Uhorchak JM., et al. "Injury of the Anterior Cruciate Ligament: A Prospective Four-Year Evaluation of 859 West Point Cadets". The American Journal of Sports Medicine 31 (2003): 831-842.

30. Swanik CB., et al. "Reestablishing proprioception and neuromuscular control in the ACL-injured athlete". Journal of Sport Rehabilitation 6.2 (1997): 182-206.

31. Adachi N., et al. "Mechanoreceptors in the anterior cruciate ligament contribute to the joint position sense". Acta Orthopaedica Scandinavica 73.3 (2002): 330-334. 
32. Dhillon MS., et al. "Differences among mechanoreceptors in healthy and injured anterior cruciate ligaments and their clinical importance". Muscles, Ligaments and Tendons Journal 2.1 (2012): 38.

33. Kraeutler MJ., et al. "Anatomy and biomechanics of the native and reconstructed anterior cruciate ligament: surgical implications". Journal of Bone and Joint Surgery 99.5 (2017): 438445.

34. Kaplan DJ and Jazrawi LM. "Secondary stabilizers of tibial rotation in the intact and anterior cruciate ligament deficient knee". Clinics in Sports Medicine 37.1 (2018): 49-59.

35. Tsoukas D., et al. "No difference in osteoarthritis after surgical and non-surgical treatment of ACL-injured knees after 10 years". Knee Surgery, Sports Traumatology, Arthroscopy 24.9 (2016): 2953-2959.

36. Barenius B., et al. "Increased risk of osteoarthritis after anterior cruciate ligament reconstruction: a 14-year follow-up study of a randomized controlled trial". The American Journal of Sports Medicine 42.5 (2014): 1049-1057.

37. Bali K., et al. "Efficacy of immunohistological methods in detecting functionally viable mechanoreceptors in the remnant stumps of injured anterior cruciate ligaments and its clinical importance". Knee Surgery, Sports Traumatology, Arthroscopy 20.1 (2012): 75-80.

\section{Assets from publication with us}

- Prompt Acknowledgement after receiving the article

- Thorough Double blinded peer review

- Rapid Publication

- Issue of Publication Certificate

- High visibility of your Published work

Website: www.actascientific.com/ Submit Article: www.actascientific.com/submission.php Email us: editor@actascientific.com

Contact us: +919182824667

Citation: Mark Daniel Wilson., et al. "The Extrinsic and Intrinsic Factors Predisposing to ACL Injuries in Female Athletes - Sports Medicine Implications in 2021". Acta Scientific Orthopaedics 4.5 (2021): 11-19. 